\title{
Buckling of Nonprismatic Column on Varying Elastic Foundation with Arbitrary Boundary Conditions
}

\author{
Ahmad A. Ghadban, ${ }^{1}$ Ahmed H. Al-Rahmani, ${ }^{2}$ \\ Hayder A. Rasheed, ${ }^{1}$ and Mohammed T. Albahttiti ${ }^{1}$ \\ ${ }^{1}$ Department of Civil Engineering, Kansas State University, Manhattan, KS 66506, USA \\ ${ }^{2}$ Smislova, Kehnemui \& Associates, PA, Potomac, MD 20854, USA \\ Correspondence should be addressed to Hayder A. Rasheed; hayder@ksu.edu
}

Received 25 August 2016; Accepted 31 October 2016; Published 27 April 2017

Academic Editor: George Tsiatas

Copyright (C) 2017 Ahmad A. Ghadban et al. This is an open access article distributed under the Creative Commons Attribution License, which permits unrestricted use, distribution, and reproduction in any medium, provided the original work is properly cited.

\begin{abstract}
Buckling of nonprismatic single columns with arbitrary boundary conditions resting on a nonuniform elastic foundation may be considered as the most generalized treatment of the subject. The buckling differential equation for such columns is extremely difficult to solve analytically. Thus, the authors propose a numerical approach by discretizing the column into a finite number of segments. Each segment has constants $E$ (modulus of elasticity), $I$ (moment of inertia), and $\beta$ (subgrade stiffness). Next, an exact analytical solution is derived for each prismatic segment resting on uniform elastic foundation. These segments are then assembled in a matrix from which the critical buckling load is obtained. The derived formulation accounts for different end boundary conditions. Validation is performed by benchmarking the present results against analytical solutions found in the literature, showing excellent agreement. After validation, more examples are solved to illustrate the power and flexibility of the proposed method. Overall, the proposed method provides reasonable results, and the examples solved demonstrate the versatility of the developed approach and some of its many possible applications.
\end{abstract}

\section{Introduction}

By the beginning of the twentieth century, many researchers got into studying the specific cases of bucking using various methods such as continuous and lumped matrix analysis, Finite Element Method (FEM), and Boundary Element Method (BEM) [1]. Dinnik reported the exact solution of simply supported columns with monomial variation in stiffness and axial load in his paper published in 1932 [2]. Simply supported tapered columns were studied and the analytical solutions were presented by Gere and Carter in 1962 [3]. In 1970, Gallagher and Lee solved an axially loaded column with a variable flexural stiffness using Finite Element Method [2]. Additionally, Elishakoff and Bert in 1988 succeeded at improving Rayleigh's method and obtaining an approximate solution for columns with variable stiffness [2]. A year later, Eisenberger presented the exact buckling solution for columns with variable cross-sections and variable axial load using any polynomial variation and using different boundary conditions [2]. In 1986, Ermopoulos published the solution for buckling of tapered bars under stepped axial loads [4]. Analysis on the buckling of uniform columns with different supports was performed by Iyengar in his book which was published in 1988 [5]. Elastic stability of elastically supported columns under the effect of distributed forces was studied by Lee and Kuo in 1991 [5]. In the same year, Arbabei and Li presented the solution for the buckling of nonprismatic elastic columns [6]. A more recent solution for the exact buckling of constrained stepped columns was presented by Yang and Park in 2003 [7]. Coşkun and Atay analyzed the critical buckling load for elastic columns of constant and variable cross-sections using variational iteration method in 2009 [6]. This variational iteration method produces an approximate solution for the presented problem. In 2012, Huang and Li published their solution for analytically determining the exact critical buckling loads of nonuniform columns [8]. Lee et al. published their calculations of natural frequencies and buckling loads for columns with intermediate multiple elastic 


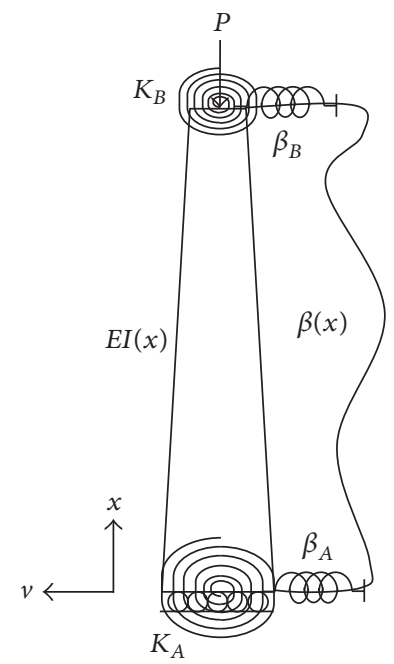

FIGURE 1: Nonprismatic columns with arbitrary boundary conditions resting on nonuniform elastic foundation.

springs in 2002 [9]. Atanackovic and Novakovic worked on Lagrange problem, in which they determined the optimal shape of an elastic column on elastic foundation using an area varying equation to represent the column shape [10]. Using Pontryagin's maximum principle, Levy presented the optimal shape of simply supported columns on elastic foundations in 1990 [10]. Using Galerkin's method, Lacarbonara solved for buckling and postbuckling of nonuniform nonlinearly elastic rods [11]. To the authors' best knowledge, this is the first paper that provides a numerical treatment combining the buckling of nonprismatic columns resting on nonuniform elastic foundation with arbitrary end boundary conditions.

\section{Formulation}

2.1. The Buckling Equation. The buckling differential equation for the column shown in Figure 1 can be derived using the bifurcation method resulting in the following equation:

$$
E I \frac{\partial^{4} v}{\partial x^{4}}+2 \frac{\partial(E I)}{\partial x} \frac{\partial^{3} v}{\partial x^{3}}+\left[\frac{\partial^{2}(E I)}{\partial x^{2}}+P\right] \frac{\partial^{2} v}{\partial x^{2}}+\beta v=0
$$

The boundary conditions (BCs) are as follows:

(1) Shear

$$
\begin{aligned}
& V(0)=-\beta_{A} v(0), \\
& V(L)=\beta_{B} v(L) .
\end{aligned}
$$

(2) Moment

$$
\begin{aligned}
& M(0)=K_{A} v^{\prime}(0), \\
& M(L)=-K_{B} v^{\prime}(L),
\end{aligned}
$$

where $E(x)$ is the column elastic modulus function, $I(x)$ is the column moment of inertia function, $P$ is the column axial load, $\beta(x)$ is the elastic foundation spring stiffness function, $v(x)$ is the column lateral deflection function, $x$ is the distance

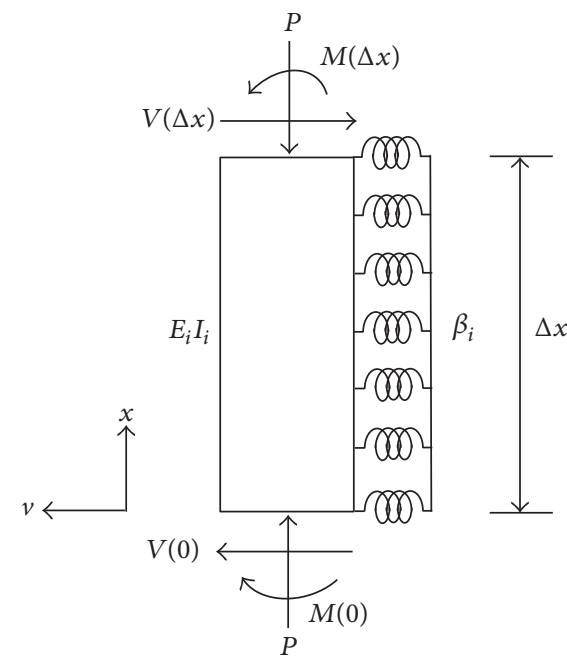

FIGURE 2: Idealized prismatic column segment resting on uniform elastic foundation.

from the bottom of the column, $\beta_{A}$ is the bottom lateral discrete spring stiffness, $\beta_{B}$ is the top lateral discrete spring stiffness, $K_{A}$ is the bottom rotational discrete spring stiffness, $K_{B}$ is the top rotational discrete spring stiffness, $L$ is the length of the column, $V(x)$ is the column shear force function, and $M(x)$ is the column moment function.

Such a differential equation can prove to be extremely difficult to solve analytically for arbitrary $E, I$, and $\beta$ functions. The authors are not aware of any analytical mathematical method that can solve this differential equation. Thus, the authors decided to solve the differential equation semianalytically by discretizing the column into a finite number of segments. Each segment has constants $E, I$, and $\beta$, as shown in Figure 2. $E, I$, and $\beta$ values for each segment were taken to be the average of each function within that segment of the column. The averages were calculated using the general equation for averaging functions within an interval:

$$
f_{\mathrm{ave}}=\frac{\int_{a}^{b} f(x) d x}{b-a} .
$$

An exact solution was then obtained for each segment using the classical buckling equation of columns on an elastic foundation. The domain of $x$ for each segment was taken to be $0 \leq x \leq \Delta x$ :

$$
\begin{aligned}
\frac{\partial^{4} v_{i}}{\partial x^{4}}+k_{i}^{2} \frac{\partial^{2} v_{i}}{\partial x^{2}}+\widehat{\beta}_{i}^{2} v_{i} & =0, \quad 0 \leq x \leq \Delta x, \\
k_{i} & =\sqrt{\frac{P}{E_{i} I_{i}}}, \\
\widehat{\beta}_{i} & =\sqrt{\frac{\beta_{i}}{E_{i} I_{i}}}, \\
\Delta x & =\frac{L}{N},
\end{aligned}
$$

where $N$ is number of segments, $\Delta x$ is segment length, and $i$ is segment number. 
2.2. Analytical Solution of the Segment Buckling Equation. The general solution to the segment differential equation with arbitrary boundary conditions is complicated and requires the consideration of four different cases. The solution to each case is shown below.

Case $1\left(\beta_{i}=0\right)$. The segment differential equation becomes

$$
\frac{\partial^{4} v_{i}}{\partial x^{4}}+k_{i}^{2} \frac{\partial^{2} v_{i}}{\partial x^{2}}=0, \quad 0 \leq x \leq \Delta x .
$$

Let $v_{i}=e^{\lambda_{i} x}$, which can be substituted into (6) resulting in

$$
\begin{aligned}
\left(\lambda_{i}^{4}+k_{i}^{2} \lambda_{i}^{2}\right) e^{\lambda_{i} x} & =0 \stackrel{\text { yields }}{\longrightarrow} \\
\lambda_{i_{1,2}} & =0, \\
\lambda_{i_{3,4}} & = \pm j k_{i},
\end{aligned}
$$

where

$$
j=\sqrt{-1} .
$$

The deflection function is then obtained as follows:

$$
v_{i}(x)=A_{i} \cos k_{i} x+B_{i} \sin k_{i} x+C_{i} x+D_{i} .
$$

Case $2\left(k_{i}^{4}-4 \widehat{\beta}_{i}^{2}=0\right)$. Let $v_{i}=e^{\lambda_{i} x}$, which can be substituted into (5) resulting in

$$
\begin{aligned}
\left(\lambda_{i}^{4}+k_{i}^{2} \lambda_{i}^{2}+\widehat{\beta}_{i}^{2}\right) e^{\lambda_{i} x} & =0 \stackrel{\text { yields }}{\longrightarrow} \\
\lambda_{i_{1,2,3,4}} & = \pm j \frac{k_{i}}{\sqrt{2}} .
\end{aligned}
$$

The deflection function is then found to be

$$
\begin{aligned}
v_{i}(x)= & A_{i} \cos \frac{k_{i} x}{\sqrt{2}}+B_{i} \sin \frac{k_{i} x}{\sqrt{2}}+C_{i} x \cos \frac{k_{i} x}{\sqrt{2}} \\
& +D_{i} x \sin \frac{k_{i} x}{\sqrt{2}} .
\end{aligned}
$$

Case $3\left(k_{i}^{4}-4 \widehat{\beta}_{i}^{2}>0\right)$. Let $v_{i}=e^{\lambda_{i} x}$, which can be substituted into (5) resulting in

$$
\begin{aligned}
\left(\lambda_{i}^{4}+k_{i}^{2} \lambda_{i}^{2}+\widehat{\beta}_{i}^{2}\right) e^{\lambda_{i} x} & =0 \stackrel{\text { yields }}{\longrightarrow} \\
\lambda_{i}^{2} & =\frac{-k_{i}^{2} \pm \sqrt{k_{i}^{4}-4 \widehat{\beta}_{i}^{2}}}{2} \stackrel{\text { yields }}{\longrightarrow} \\
\lambda_{i_{1,2}} & = \pm j \alpha_{1 i}, \\
\lambda_{i_{3,4}} & = \pm j \alpha_{2 i},
\end{aligned}
$$

where

$$
\begin{aligned}
& \alpha_{1 i}=\sqrt{\frac{k_{i}^{2}+\sqrt{k_{i}^{4}-4 \widehat{\beta}_{i}^{2}}}{2}}, \\
& \alpha_{2 i}=\sqrt{\frac{k_{i}^{2}-\sqrt{k_{i}^{4}-4 \widehat{\beta}_{i}^{2}}}{2}} .
\end{aligned}
$$

The deflection function is then obtained to be as follows

$$
\begin{aligned}
v_{i}(x)= & A_{i} \cos \alpha_{1 i} x+B_{i} \sin \alpha_{1 i} x+C_{i} \cos \alpha_{2 i} x \\
& +D_{i} \sin \alpha_{2 i} x .
\end{aligned}
$$

Case $4\left(k_{i}^{4}-4 \widehat{\beta}_{i}^{2}<0\right)$. Let $v_{i}=e^{\lambda_{i} x}$, which can be substituted into (5) resulting in

$$
\begin{aligned}
\left(\lambda_{i}^{4}+k_{i}^{2} \lambda_{i}^{2}+\widehat{\beta}_{i}^{2}\right) e^{\lambda_{i} x} & =0 \stackrel{\text { yields }}{\longrightarrow} \\
\lambda_{i}^{2} & =\frac{-k_{i}^{2} \pm \sqrt{k_{i}^{4}-4 \widehat{\beta}_{i}^{2}}}{2} \\
& =\frac{-k_{i}^{2}}{2} \pm j \frac{\sqrt{4 \widehat{\beta}_{i}^{2}-k_{i}^{4}}}{2} .
\end{aligned}
$$

Let $\lambda_{i}=a_{i}+j b_{i}$ implying $\lambda_{i}^{2}=\left(a_{i}^{2}-b_{i}^{2}\right)+j 2 a_{i} b_{i}$ which results in the following two equations with two unknowns, $a_{i}$ and $b_{i}$ :

$$
\begin{aligned}
a_{i}^{2}-b_{i}^{2} & =\frac{-k_{i}^{2}}{2}, \\
2 a_{i} b_{i} & = \pm \frac{\sqrt{4 \widehat{\beta}_{i}^{2}-k_{i}^{4}}}{2} .
\end{aligned}
$$

From (17) we get

$$
\begin{aligned}
a_{i} & = \pm \frac{\sqrt{4 \widehat{\beta}_{i}^{2}-k_{i}^{4}}}{4 b_{i}} \stackrel{\text { yields }}{\longrightarrow} \\
a_{i}^{2} & =\frac{4 \widehat{\beta}_{i}^{2}-k_{i}^{4}}{16 b_{i}^{2}} .
\end{aligned}
$$

Substituting into (16) we obtain

$$
\begin{aligned}
\frac{4 \widehat{\beta}_{i}^{2}-k_{i}^{4}}{16 b_{i}^{2}}-b_{i}^{2} & =\frac{-k_{i}^{2}}{2} \stackrel{\text { yields }}{\longrightarrow} \\
16 b_{i}^{4}-8 k_{i}^{2} b_{i}^{2}+k_{i}^{4}-4 \widehat{\beta}_{i}^{2} & =0 \stackrel{\text { yields }}{\longrightarrow} \\
b_{i}^{2} & =\frac{k_{i}^{2} \pm 2 \widehat{\beta}_{i}}{4} .
\end{aligned}
$$

We know that $k_{i}^{4}-4 \widehat{\beta}_{i}^{2}<0 \stackrel{\text { yields }}{\longrightarrow}\left(k_{i}^{2}+2 \widehat{\beta}_{i}\right)\left(k_{i}^{2}-2 \widehat{\beta}_{i}\right)<0$, but it is obvious that $k_{i}^{2}+2 \widehat{\beta}_{i}>0$. Therefore, $k_{i}^{2}-2 \widehat{\beta}_{i}<0 \stackrel{\text { yields }}{\longrightarrow}$ $b_{i}^{2} \neq\left(k_{i}^{2}-2 \widehat{\beta}_{i}\right) / 4$ since $b_{i}^{2}>0$. This leaves the following: $b_{i}^{2}=\left(k_{i}^{2}+2 \widehat{\beta}_{i}\right) / 4 \stackrel{\text { yields }}{\longrightarrow} b_{i}= \pm \sqrt{2 \widehat{\beta}_{i}+k_{i}^{2}} / 2$.

Using (17) we can then obtain $a_{i}$ as follows:

$$
a_{i}= \pm \frac{\sqrt{2 \widehat{\beta}_{i}-k_{i}^{2}}}{2} .
$$


Therefore, $\lambda_{i}$ is found to be

$$
\begin{aligned}
\lambda_{i} & = \pm \frac{\sqrt{2 \widehat{\beta}_{i}-k_{i}^{2}}}{2} \pm j \frac{\sqrt{2 \widehat{\beta}_{i}+k_{i}^{2}}}{2} \stackrel{\text { yields }}{\longrightarrow} \\
\lambda_{i_{1,2}} & =\gamma_{1 i} \pm j g_{i}, \\
\lambda_{i_{3,4}} & =\gamma_{2 i} \pm j g_{i},
\end{aligned}
$$

where

$$
\begin{aligned}
g_{i} & =\frac{\sqrt{2 \widehat{\beta}_{i}+k_{i}^{2}}}{2}, \\
\gamma_{1 i} & =\frac{\sqrt{2 \widehat{\beta}_{i}-k_{i}^{2}}}{2}, \\
\gamma_{2 i} & =\frac{-\sqrt{2 \widehat{\beta}_{i}-k_{i}^{2}}}{2} .
\end{aligned}
$$

The deflection function is then obtained to be

$$
\begin{aligned}
v_{i}(x)= & A_{i} e^{\gamma_{1 i} x} \cos g_{i} x+B_{i} e^{\gamma_{1 i} x} \sin g_{i} x \\
& +C_{i} e^{\gamma_{2 i} x} \cos g_{i} x+D_{i} e^{\gamma_{2 i} x} \sin g_{i} x
\end{aligned}
$$

where $A_{i}, B_{i}, C_{i}$, and $D_{i}$ are the constant coefficients for each segment.

After solving the segment differential equation, the following compatibility conditions were established at each node between the segments.

(1) Displacement

$$
\begin{aligned}
v_{i}(0) & =v_{i-1}(\Delta x), \\
v_{i}(\Delta x) & =v_{i+1}(0) .
\end{aligned}
$$

(2) Slope

$$
\begin{aligned}
v_{i}^{\prime}(0) & =v_{i-1}^{\prime}(\Delta x), \\
v_{i}^{\prime}(\Delta x) & =v_{i+1}^{\prime}(0) .
\end{aligned}
$$

(3) Moment

$$
\begin{gathered}
E_{i} I_{i} v_{i}^{\prime \prime}(0)=E_{i-1} I_{i-1} v_{i-1}^{\prime \prime}(\Delta x), \\
E_{i} I_{i} v_{i}^{\prime \prime}(\Delta x)=E_{i+1} I_{i+1} v_{i+1}^{\prime \prime}(0) .
\end{gathered}
$$

(4) Shear

$$
\begin{aligned}
& E_{i} I_{i} v_{i}^{\prime \prime \prime}(0)+P v_{i}^{\prime}(0) \\
& \quad=E_{i-1} I_{i-1} v_{i-1}^{\prime \prime \prime}(\Delta x)+P v_{i-1}^{\prime}(\Delta x),
\end{aligned}
$$

$$
\begin{aligned}
& E_{i} I_{i} v_{i}^{\prime \prime \prime}(\Delta x)+P v_{i}^{\prime}(\Delta x) \\
& \quad=E_{i+1} I_{i+1} v_{i+1}^{\prime \prime \prime}(0)+P v_{i+1}^{\prime}(0) .
\end{aligned}
$$

Finally, in order to obtain the coefficient matrix $(P$ matrix), the following boundary conditions were established.

(1) Shear

$$
\begin{aligned}
E_{1} I_{1} v_{1}^{\prime \prime \prime}(0)+P v_{1}^{\prime}(0) & =-\beta_{A} v_{1}(0), \\
E_{N} I_{N} v_{N}^{\prime \prime \prime}(\Delta x)+P v_{N}^{\prime}(\Delta x) & =\beta_{B} v_{N}(\Delta x) .
\end{aligned}
$$

(2) Moment

$$
\begin{gathered}
E_{1} I_{1} v_{1}^{\prime \prime}(0)=K_{A} v_{1}^{\prime}(0), \\
E_{N} I_{N} v_{N}^{\prime \prime}(\Delta x)=-K_{B} v_{N}^{\prime}(\Delta x) .
\end{gathered}
$$

Solving the following system of equations would then render the buckling load:

$$
[P]\{C\}=\{0\},
$$

where $P$ is the $P$-Matrix and $C=$

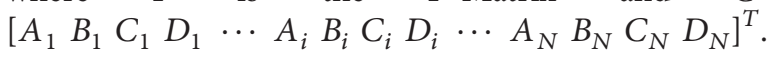

2.3. The P-Matrix. The structure of the $P$-matrix is as follows.

(1) One Segment

$[4 \times 4$ BC Matrix]

(2) Two Segments 
(3) Three Segments

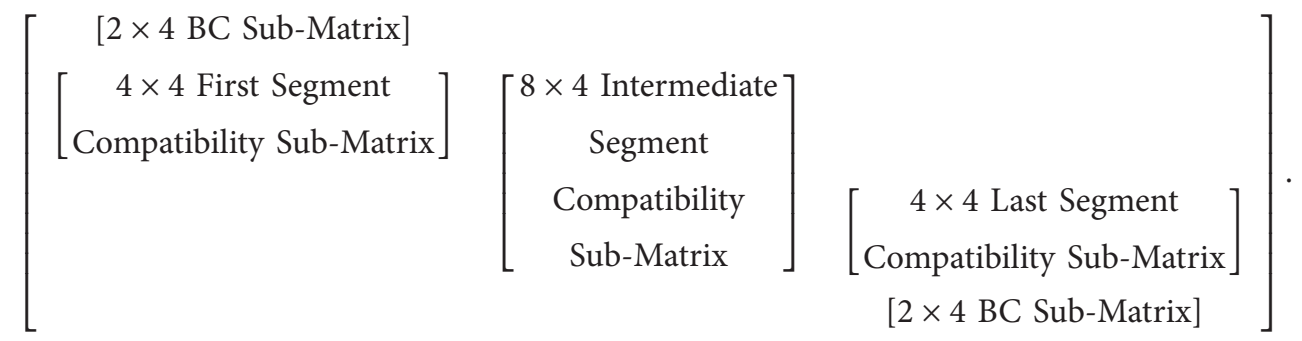

For the case of an assembly with more than three segments, the structure of the $P$-matrix would be the same as the three segments case but with additional intermediate segment compatibility submatrices that are shifted diagonally downwards. The buckling load would then be the load that produces a singular $P$-matrix.

The submatrices inside the $P$-matrix are obtained by applying the compatibility and boundary conditions and are dependent on the case that governs each segment, as explained earlier. These submatrices were found to be as shown below.

\section{(a) Intermediate Segments}

Case $2\left(k_{i}^{4}-4 \widehat{\beta}_{i}^{2}=0\right)$.
Case $1\left(\beta_{i}=0\right)$.

$$
\left[\begin{array}{cccc}
1 & 0 & 0 & 1 \\
0 & k_{i} & 1 & 0 \\
-P & 0 & 0 & 0 \\
0 & 0 & P & 0 \\
-\cos k_{i} \Delta x & -\sin k_{i} \Delta x & -\Delta x & -1 \\
k_{i} \sin k_{i} \Delta x & -k_{i} \cos k_{i} \Delta x & -1 & 0 \\
P \cos k_{i} \Delta x & P \sin k_{i} \Delta x & 0 & 0 \\
0 & 0 & -P & 0
\end{array}\right] .
$$

$$
\left[\begin{array}{cccc}
1 & 0 & 0 & 0 \\
0 & \frac{k_{i}}{\sqrt{2}} & 1 & 0 \\
\frac{-P}{2} & 0 & 0 & \sqrt{2} E_{i} I_{i} k_{i} \\
0 & \frac{k_{i} P}{2 \sqrt{2}} & \frac{-P}{2} & 0 \\
-\cos \frac{k_{i} \Delta x}{\sqrt{2}} & -\sin \frac{k_{i} \Delta x}{\sqrt{2}} & -\Delta x \cos \frac{k_{i} \Delta x}{\sqrt{2}} & -\Delta x \sin \frac{k_{i} \Delta x}{\sqrt{2}} \\
\frac{k_{i}}{\sqrt{2}} \sin \frac{k_{i} \Delta x}{\sqrt{2}} & -\frac{k_{i}}{\sqrt{2}} \cos \frac{k_{i} \Delta x}{\sqrt{2}} & \Delta x \frac{k_{i}}{\sqrt{2}} \sin \frac{k_{i} \Delta x}{\sqrt{2}}-\cos \frac{k_{i} \Delta x}{\sqrt{2}} & -\Delta x \frac{k_{i}}{\sqrt{2}} \cos \frac{k_{i} \Delta x}{\sqrt{2}}-\sin \frac{k_{i} \Delta x}{\sqrt{2}} \\
\frac{P}{2} \cos \frac{k_{i} \Delta x}{\sqrt{2}} & \frac{P}{2} \sin \frac{k_{i} \Delta x}{\sqrt{2}} & \Delta x \frac{P}{2} \cos \frac{k_{i} \Delta x}{\sqrt{2}}+\sqrt{2} E_{i} I_{i} k_{i} \sin \frac{k_{i} \Delta x}{\sqrt{2}} & \Delta x \frac{P}{2} \sin \frac{k_{i} \Delta x}{\sqrt{2}}-\sqrt{2} E_{i} I_{i} k_{i} \cos \frac{k_{i} \Delta x}{\sqrt{2}} \\
\frac{k_{i} P}{2 \sqrt{2}} \sin \frac{k_{i} \Delta x}{\sqrt{2}} & \frac{-k_{i} P}{2 \sqrt{2}} \cos \frac{k_{i} \Delta x}{\sqrt{2}} & \Delta x \frac{k_{i} P}{2 \sqrt{2}} \sin \frac{k_{i} \Delta x}{\sqrt{2}}+\frac{P}{2} \cos \frac{k_{i} \Delta x}{\sqrt{2}} & -\Delta x \frac{k_{i} P}{2 \sqrt{2}} \cos \frac{k_{i} \Delta x}{\sqrt{2}}+\frac{P}{2} \sin \frac{k_{i} \Delta x}{\sqrt{2}}
\end{array}\right]
$$


Case $3\left(k_{i}^{4}-4 \widehat{\beta}_{i}^{2}>0\right)$.

$$
\left[\begin{array}{cccc}
1 & 0 & 1 & 0 \\
0 & \alpha_{1 i} & 0 & \alpha_{2 i} \\
-E_{i} I_{i} \alpha_{1 i}^{2} & 0 & -E_{i} I_{i} \alpha_{2 i}^{2} & 0 \\
0 & P \alpha_{1 i}-E_{i} I_{i} \alpha_{1 i}^{3} & 0 & P \alpha_{2 i}-E_{i} I_{i} \alpha_{2 i}^{3} \\
-\cos \alpha_{1 i} \Delta x & -\sin \alpha_{1 i} \Delta x & -\cos \alpha_{2 i} \Delta x & -\sin \alpha_{2 i} \Delta x \\
\alpha_{1 i} \sin \alpha_{1 i} \Delta x & -\alpha_{1 i} \cos \alpha_{1 i} \Delta x & \alpha_{2 i} \sin \alpha_{2 i} \Delta x & -\alpha_{2 i} \cos \alpha_{2 i} \Delta x \\
E_{i} I_{i} \alpha_{1 i}^{2} \cos \alpha_{1 i} \Delta x & E_{i} I_{i} \alpha_{1 i}^{2} \sin \alpha_{1 i} \Delta x & E_{i} I_{i} \alpha_{2 i}^{2} \cos \alpha_{2 i} \Delta x & E_{i} I_{i} \alpha_{2 i}^{2} \sin \alpha_{2 i} \Delta x \\
\left(P \alpha_{1 i}-E_{i} I_{i} \alpha_{1 i}^{3}\right) \sin \alpha_{1 i} \Delta x & -\left(P \alpha_{1 i}-E_{i} I_{i} \alpha_{1 i}^{3}\right) \cos \alpha_{1 i} \Delta x & \left(P \alpha_{2 i}-E_{i} I_{i} \alpha_{2 i}^{3}\right) \sin \alpha_{2 i} \Delta x & -\left(P \alpha_{2 i}-E_{i} I_{i} \alpha_{2 i}^{3}\right) \cos \alpha_{2 i} \Delta x
\end{array}\right]
$$

Case $4\left(k_{i}^{4}-4 \widehat{\beta}_{i}^{2}<0\right)$.

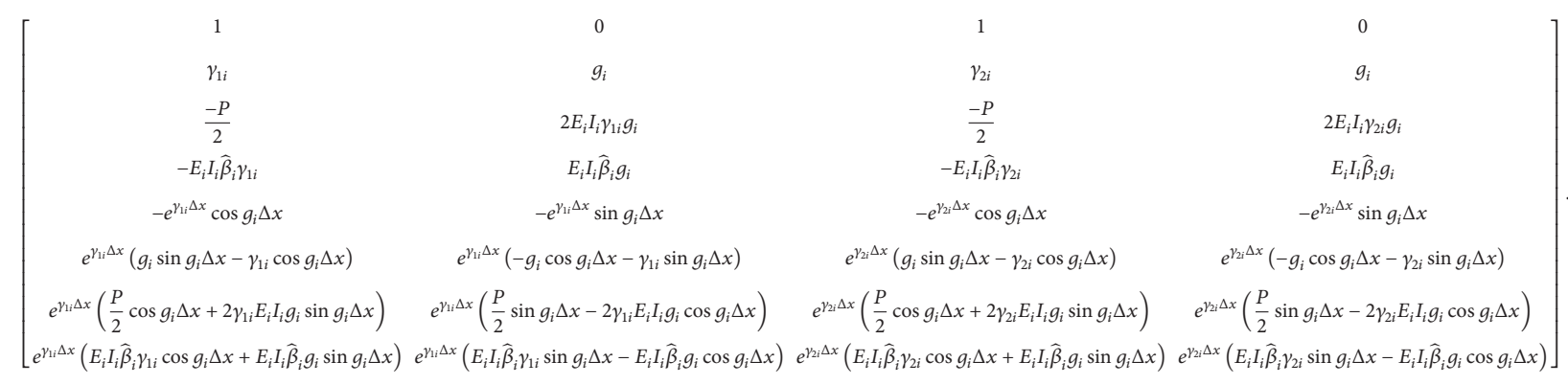

(b) First Segment

Case $1\left(\beta_{1}=0\right)$.

$$
\left[\begin{array}{cccc}
\beta_{A} & 0 & P & \beta_{A} \\
-P & -K_{A} k_{1} & -K_{A} & 0 \\
-\cos k_{1} \Delta x & -\sin k_{1} \Delta x & -\Delta x & -1 \\
k_{1} \sin k_{1} \Delta x & -k_{1} \cos k_{1} \Delta x & -1 & 0 \\
P \cos k_{1} \Delta x & P \sin k_{1} \Delta x & 0 & 0 \\
0 & 0 & -P & 0
\end{array}\right] .
$$

Case $2\left(k_{1}^{4}-4 \widehat{\beta}_{1}^{2}=0\right)$.

$$
\left[\begin{array}{cccc}
\beta_{A} & \frac{k_{1} P}{2 \sqrt{2}} & -\frac{P}{2} & 0 \\
-\frac{P}{2} & -K_{A} \frac{k_{1}}{\sqrt{2}} & -K_{A} & \sqrt{2} E_{1} I_{1} k_{1} \\
-\cos \frac{k_{1} \Delta x}{\sqrt{2}} & -\sin \frac{k_{1} \Delta x}{\sqrt{2}} & -\Delta x \cos \frac{k_{1} \Delta x}{\sqrt{2}} & -\Delta x \sin \frac{k_{1} \Delta x}{\sqrt{2}} \\
\frac{k_{1}}{\sqrt{2}} \sin \frac{k_{1} \Delta x}{\sqrt{2}} & -\frac{k_{1}}{\sqrt{2} \cos \frac{k_{1} \Delta x}{\sqrt{2}}} & \Delta x \frac{k_{1}}{\sqrt{2}} \sin \frac{k_{1} \Delta x}{\sqrt{2}}-\cos \frac{k_{1} \Delta x}{\sqrt{2}} & -\Delta x \frac{k_{1}}{\sqrt{2}} \cos \frac{k_{1} \Delta x}{\sqrt{2}}-\sin \frac{k_{1} \Delta x}{\sqrt{2}} \\
\frac{P}{2} \cos \frac{k_{1} \Delta x}{\sqrt{2}} & \frac{P}{2} \sin \frac{k_{1} \Delta x}{\sqrt{2}} & \Delta x \frac{P}{2} \cos \frac{k_{1} \Delta x}{\sqrt{2}}+\sqrt{2} E_{1} I_{1} k_{1} \sin \frac{k_{1} \Delta x}{\sqrt{2}} & \Delta x \frac{P}{2} \sin \frac{k_{1} \Delta x}{\sqrt{2}}-\sqrt{2} E_{1} I_{1} k_{1} \cos \frac{k_{1} \Delta x}{\sqrt{2}} \\
\frac{k_{1} P}{2 \sqrt{2}} \sin \frac{k_{1} \Delta x}{\sqrt{2}} & \frac{-k_{1} P}{2 \sqrt{2}} \cos \frac{k_{1} \Delta x}{\sqrt{2}} & \Delta x \frac{k_{1} P}{2 \sqrt{2}} \sin \frac{k_{1} \Delta x}{\sqrt{2}}+\frac{P}{2} \cos \frac{k_{1} \Delta x}{\sqrt{2}} & -\Delta x \frac{k_{1} P}{2 \sqrt{2}} \cos \frac{k_{1} \Delta x}{\sqrt{2}}+\frac{P}{2} \sin \frac{k_{1} \Delta x}{\sqrt{2}}
\end{array}\right] .
$$


Case $3\left(k_{1}^{4}-4 \widehat{\beta}_{1}^{2}>0\right)$.

$\left[\begin{array}{cccc}\beta_{A} & P \alpha_{11}-E_{1} I_{1} \alpha_{11}^{3} & \beta_{A} & P \alpha_{21}-E_{1} I_{1} \alpha_{21}^{3} \\ -E_{1} I_{1} \alpha_{11}^{2} & -K_{A} \alpha_{11} & -E_{1} I_{1} \alpha_{21}^{2} & -K_{A} \alpha_{21} \\ -\cos \alpha_{11} \Delta x & -\sin \alpha_{11} \Delta x & -\cos \alpha_{21} \Delta x & -\sin \alpha_{21} \Delta x \\ \alpha_{11} \sin \alpha_{11} \Delta x & -\alpha_{11} \cos \alpha_{11} \Delta x & \alpha_{21} \sin \alpha_{21} \Delta x & -\alpha_{21} \cos \alpha_{21} \Delta x \\ E_{1} I_{1} \alpha_{11}^{2} \cos \alpha_{11} \Delta x & E_{1} I_{1} \alpha_{11}^{2} \sin \alpha_{11} \Delta x & E_{1} I_{1} \alpha_{21}^{2} \cos \alpha_{21} \Delta x & E_{1} I_{1} \alpha_{21}^{2} \sin \alpha_{21} \Delta x \\ \left(P \alpha_{11}-E_{1} I_{1} \alpha_{11}^{3}\right) \sin \alpha_{11} \Delta x & -\left(P \alpha_{11}-E_{1} I_{1} \alpha_{11}^{3}\right) \cos \alpha_{11} \Delta x & \left(P \alpha_{21}-E_{1} I_{1} \alpha_{21}^{3}\right) \sin \alpha_{21} \Delta x & -\left(P \alpha_{21}-E_{1} I_{1} \alpha_{21}^{3}\right) \cos \alpha_{21} \Delta x\end{array}\right]$.

Case $4\left(k_{1}^{4}-4 \widehat{\beta}_{1}^{2}<0\right)$.

\begin{tabular}{|c|c|c|c|}
\hline$\beta_{A}-E_{1} I_{1} \widehat{\beta}_{1} \gamma_{11}$ & $E_{1} I_{1} \hat{\beta}_{1} g_{1}$ & $\beta_{A}-E_{1} I_{1} \hat{\beta}_{1} \gamma_{21}$ & $E_{1} I_{1} \hat{\beta}_{1} g_{1}$ \\
\hline$-K_{A} \gamma_{11}-\frac{P}{2}$ & $-\left(K_{A}-2 E_{1} I_{1} \gamma_{11}\right) g_{1}$ & $-K_{A} \gamma_{21}-\frac{P}{2}$ & $-\left(K_{A}-2 E_{1} I_{1} \gamma_{21}\right) g_{1}$ \\
\hline$-e^{\gamma_{11} \Delta x} \cos g_{1} \Delta x$ & $-e^{\gamma_{11} \Delta x} \sin g_{1} \Delta x$ & $-e^{\gamma_{21} \Delta x} \cos g_{1} \Delta x$ & $-e^{\gamma_{21} \Delta x} \sin g_{1} \Delta x$ \\
\hline$e^{\gamma_{11} \Delta x}\left(g_{1} \sin g_{1} \Delta x-\gamma_{11} \cos g_{1} \Delta x\right)$ & $e^{\gamma_{11} \Delta x}\left(-g_{1} \cos g_{1} \Delta x-\gamma_{11} \sin g_{1} \Delta x\right)$ & $e^{\gamma_{21} \Delta x}\left(g_{1} \sin g_{1} \Delta x-\gamma_{21} \cos g_{1} \Delta x\right)$ & $e^{\gamma_{21} \Delta x}\left(-g_{1} \cos g_{1} \Delta x-\gamma_{21} \sin g_{1} \Delta x\right)$ \\
\hline$e^{\gamma_{11} \Delta x}\left(\frac{P}{2} \cos g_{1} \Delta x+2 \gamma_{11} E_{1} I_{1} g_{1} \sin g_{1} \Delta x\right)$ & $e^{\gamma_{11} \Delta x}\left(\frac{P}{2} \sin g_{1} \Delta x-2 \gamma_{11} E_{1} I_{1} g_{1} \cos g_{1} \Delta x\right)$ & $e^{\gamma_{21} \Delta x}\left(\frac{P}{2} \cos g_{1} \Delta x+2 \gamma_{21} E_{1} I_{1} g_{1} \sin g_{1} \Delta x\right)$ & $e^{\gamma_{21} \Delta x}\left(\frac{P}{2} \sin g_{1} \Delta x-2 \gamma_{21} E_{1} I_{1} g_{1} \cos g_{1} \Delta x\right)$ \\
\hline
\end{tabular}

(c) Last Segment

Case $1\left(\beta_{N}=0\right)$.

$\left[\begin{array}{cccc}1 & 0 & 0 & 1 \\ 0 & k_{N} & 1 & 0 \\ -P & 0 & 0 & 0 \\ 0 & 0 & P & 0 \\ -\beta_{B} \cos k_{N} \Delta x & -\beta_{B} \sin k_{N} \Delta x & P-\beta_{B} \Delta x & -\beta_{B} \\ -P \cos k_{N} \Delta x-K_{B} k_{N} \sin k_{N} \Delta x & -P \sin k_{N} \Delta x+K_{B} k_{N} \cos k_{N} \Delta x & K_{B} & 0\end{array}\right]$.

Case $2\left(k_{N}^{4}-4 \widehat{\beta}_{N}^{2}=0\right)$.

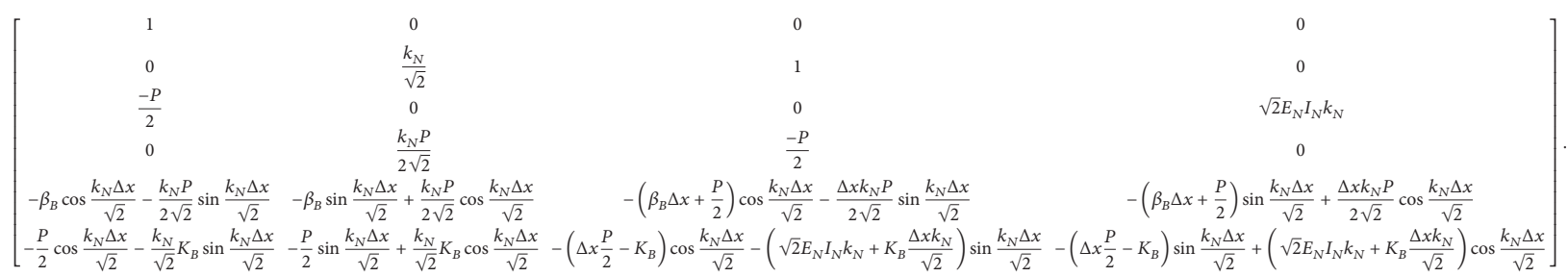


Case $3\left(k_{N}^{4}-4 \widehat{\beta}_{N}^{2}>0\right)$.

$\left[\begin{array}{cccc}1 & 0 & 1 & 0 \\ 0 & \alpha_{1 N} & -E_{N} I_{N} \alpha_{2 N}^{2} & 0 \\ -E_{N} I_{N} \alpha_{1 N}^{2} & 0 & 0 & 0 \\ 0 & P \alpha_{1 N}-E_{N} I_{N} \alpha_{1 N}^{3} & P \alpha_{2 N}-E_{N} I_{N} \alpha_{2 N}^{3} \\ -\beta_{B} \cos \alpha_{1 N} \Delta x-\left(P \alpha_{1 N}-E_{N} I_{N} \alpha_{1 N}^{3}\right) \sin \alpha_{1 N} \Delta x & -\beta_{B} \sin \alpha_{1 N} \Delta x+\left(P \alpha_{1 N}-E_{N} I_{N} \alpha_{1 N}^{3}\right) \cos \alpha_{1 N} \Delta x & -\beta_{B} \cos \alpha_{2 N} \Delta x-\left(P \alpha_{2 N}-E_{N} I_{N} \alpha_{2 N}^{3}\right) \sin \alpha_{2 N} \Delta x & -\beta_{B} \sin \alpha_{2 N} \Delta x+\left(P \alpha_{2 N}-E_{N} I_{N} \alpha_{2 N}^{3}\right) \cos \alpha_{2 N} \Delta x \\ -K_{B} \alpha_{1 N} \sin \alpha_{1 N} \Delta x-E_{N} I_{N} \alpha_{1 N}^{2} \cos \alpha_{1 N} \Delta x & K_{B} \alpha_{1 N} \cos \alpha_{1 N} \Delta x-E_{N} I_{N} \alpha_{1 N}^{2} \sin \alpha_{1 N} \Delta x & -K_{B} \alpha_{2 N} \sin \alpha_{2 N} \Delta x-E_{N} I_{N} \alpha_{2 N}^{2} \cos \alpha_{2 N} \Delta x & K_{B} \alpha_{2 N} \cos \alpha_{2 N} \Delta x-E_{N} I_{N} \alpha_{2 N}^{2} \sin \alpha_{2 N} \Delta x\end{array}\right]$.

Case $4\left(k_{N}^{4}-4 \widehat{\beta}_{N}^{2}<0\right)$.

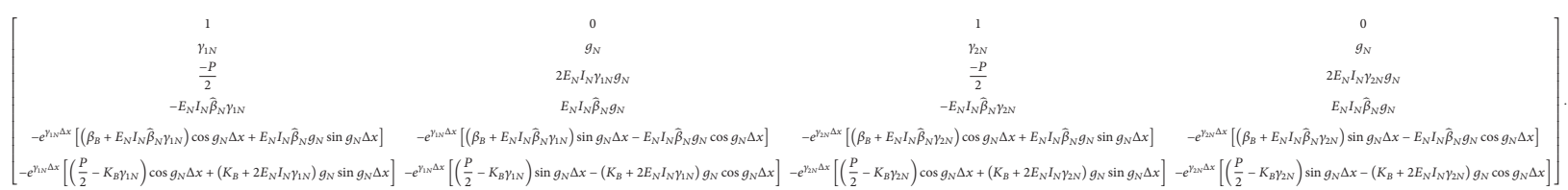

2.4. The Mode Shape. The mode shape can be obtained by substituting the buckling load into the $P$-matrix and setting one of the constant coefficients to be equal to 1 . The original system looks like the following:

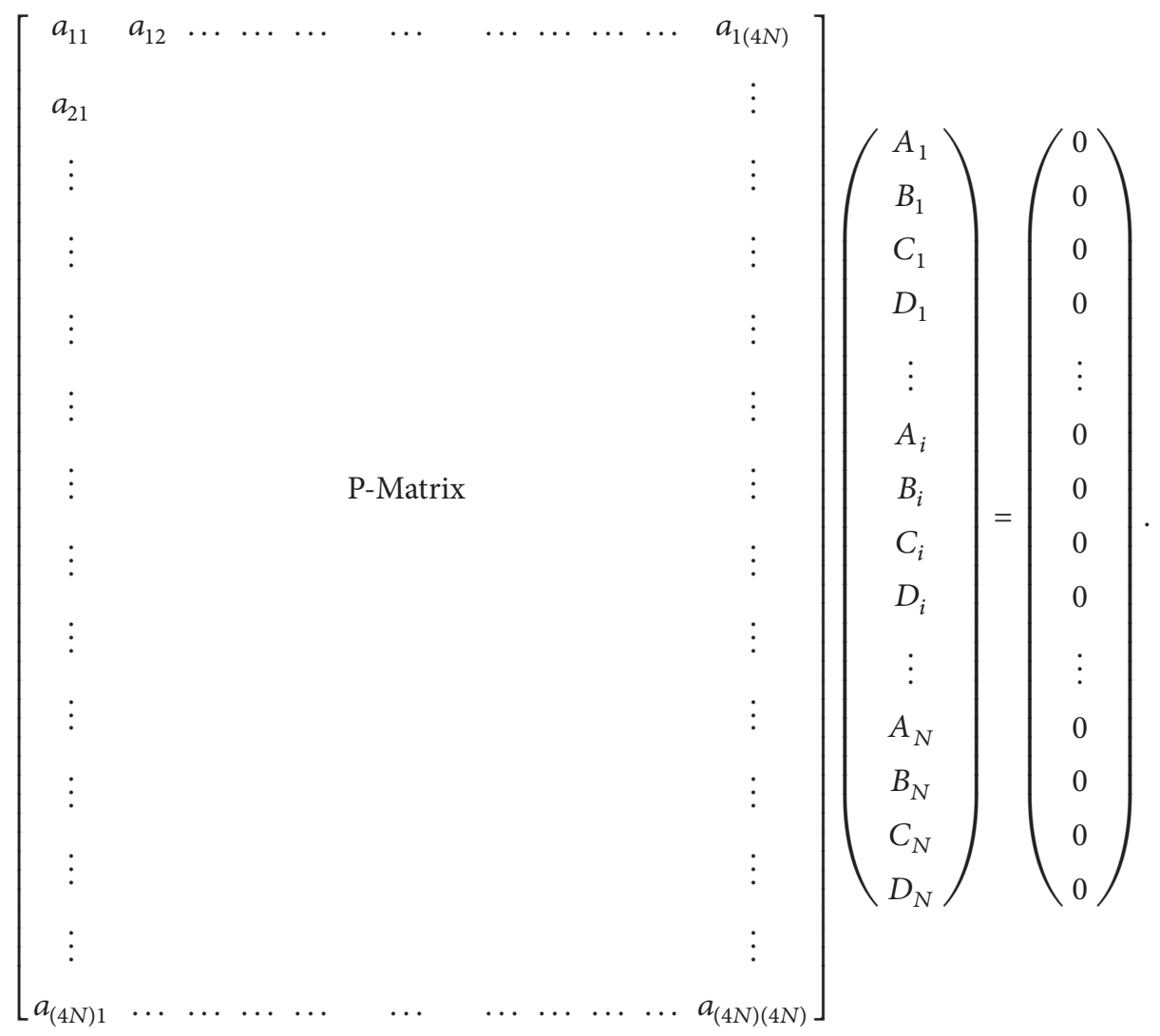


After letting $D_{N}=1$, the following system of equations is obtained.

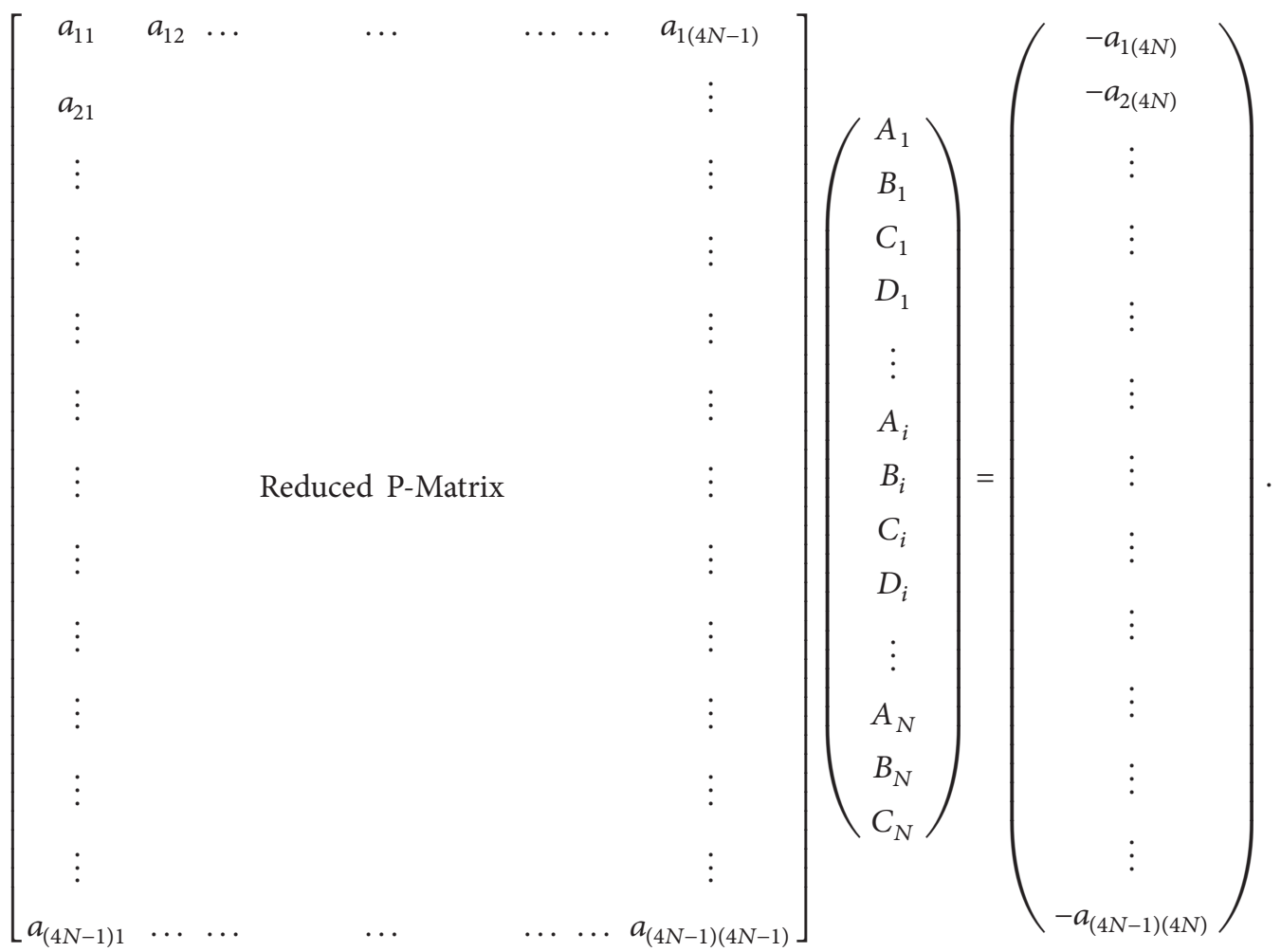

The constant coefficients can then be calculated by solving the system as follows:

$$
\left\{C_{R}\right\}=\left[P_{R}\right]^{-1}\{F\}
$$

where $P_{R}$ is the reduced $P$-matrix, $C_{R}=$ $\left[\begin{array}{lllllllllllll}A_{1} & B_{1} & C_{1} & D_{1} & \cdots & A_{i} & B_{i} & C_{i} & D_{i} & \cdots & A_{N} & B_{N} & C_{N}\end{array}\right]^{T}$, and $F=\left[\begin{array}{llllll}-a_{1(4 N)} & -a_{2(4 N)} & \cdots & \cdots & \cdots & -a_{(4 N-1)(4 N)}\end{array}\right]^{T}$.

After substituting the constants back into their respective equations, one can obtain the deflection functions and plot the mode shape.

\section{Sensitivity Analysis}

As the proposed approach solves the differential equation for buckling numerically by discretizing the column into a finite number of segments, a sensitivity analysis was conducted to determine the optimum number of segments necessary to obtain accurate results. The sensitivity analysis was conducted for a simply supported column with both cross-section and soil stiffness varying to account for all approximations involved in the solution. The following functions were used: $E I=x$ and $\beta=x^{2}$. The first 3 buckling loads and their associated mode shapes were determined. Table 1 shows the results for 10, 20, 50, 100, and 200 segments.
The results, for the discretization segments above, were reasonably close; however, the results required a high segment count to stabilize. In the next section, it will be shown that this variation is not significant as the obtained results with a segment count of 20 provided excellent agreement with the results in the literature. Figure 3 shows the first three mode shapes, which did not vary with the change in the number of segments.

\section{Validation of the Proposed Approach}

In order to validate the proposed approach, a few cases were analyzed and compared with available solutions from the literature. For the first case, a column resting on elastic foundation is analyzed. A fixed-pinned column was evaluated with the following parameters: $E I=1, L=1$, and $\beta=100$ [10]. In this case, a single element was used in the analysis as no variation in any of the parameters occurs along the span. The critical load was determined to be 28.307, which matches the load obtained by Wang and his coauthors [12]. Figure 4 shows the first three mode shapes for this case and their associated buckling loads.

The following cases are those for a nonprismatic column, as derived by Gere and Carter [13]. For Case 2, nonprismatic fixed-pinned column, as derived by Gere and Carter [13], was analyzed. The parameters were as follows: $E I=(1+$ $3.5(x / L))^{3}, L=1$, and $\beta=0$, and the number of elements 
TABLE 1: Sensitivity analysis results.

\begin{tabular}{lccc}
\hline$N$ & $P_{\mathrm{cr}}{ }^{1}$ & $P_{\mathrm{cr}}{ }^{2}$ & $P_{\mathrm{cr}}{ }^{3}$ \\
\hline 10 & 3.598021035 & 11.85057958 & 26.03460752 \\
20 & 3.655748351 & 12.00379038 & 25.20788505 \\
50 & 3.68272198 & 12.21549833 & 25.50181667 \\
100 & 3.688362459 & 12.2799962 & 25.72594058 \\
200 & 3.690129118 & 12.30323418 & 25.82633913 \\
\hline
\end{tabular}

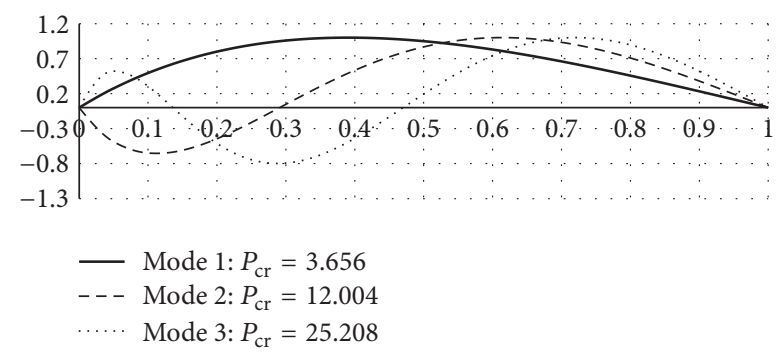

FIgURE 3: Mode shapes for column utilized in sensitivity analysis.

was taken to be 20. The buckling load obtained was 221.031, compared to 222.2 obtained by Gere's approach. Figure 5 shows the mode shape obtained for this case.

In Case 3, the variable end conditions implementation was validated. This was accomplished by analyzing cases with different rotational spring values at the ends and comparing the results with the alignment charts. This was done for both the sway permitted and sway not permitted cases. The column analyzed had the following parameters: $E=I=L=1$. For the pinned-pinned reference case, the Euler buckling load $\left(P_{E}\right)$ was obtained to be 9.8696 . The results obtained are shown in Table 2.

The results obtained showed excellent agreement with those obtained from the alignment charts. This validates the derivation of variable end supports and its implementation in the computer program.

Finally, in Cases 4 and 5, the approach was validated for cases with nonprismatic sections [2] and variable soil stiffness [14], respectively. In Case 4, columns with different boundary conditions were analyzed as shown in Table 3 . The parameters for these columns were as follows: $E I=(1+x / L), L=1$, and $\beta=0$. The results obtained are also shown in Table 3 .

For Case 5, a column resting on a variable elastic foundation was analyzed. The column had the following parameters: $E I=1.5 \times 10^{5} \mathrm{~N} \cdot \mathrm{m}^{2}, L=3 \mathrm{~m}$, and $\beta=\left(4 x-3 x^{2}+x^{3}\right) 10^{6} \mathrm{~N} /$ $\mathrm{m}^{2}$. The critical load was obtained to be $148514.92 \mathrm{~N}$, which is very close to what was obtained by Eisenberger and Clastornik [14].

\section{Examples}

5.1. Example 1: Column on Variable (Linear and Nonlinear) Elastic Foundation. After validating the proposed formulation in the previous section, the case of nonuniform soil pressure was investigated. A simply supported column was analyzed with 20 segments. The column's parameters were as follows: $E I=100$ and $L=10$, and, similar to what

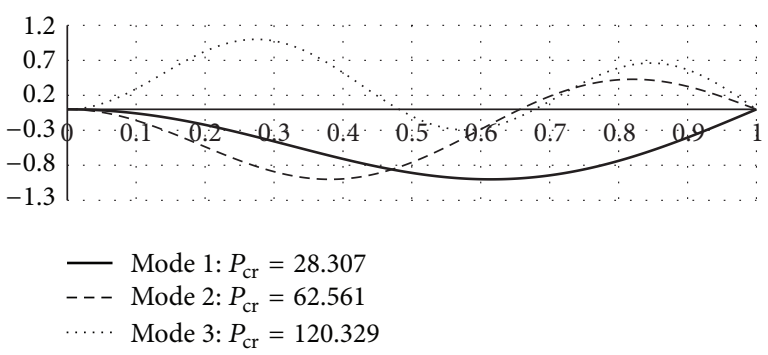

Figure 4: Mode shapes for Case 1.

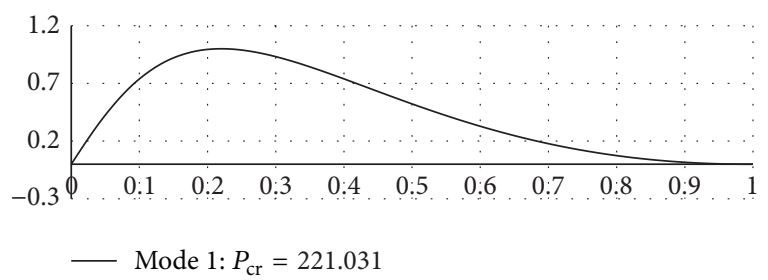

Figure 5: Mode shape for Case 2.

Gere and Carter [13] did for the moment of inertia in nonprismatic columns, the soil stiffness $(\beta)$ was taken to be as follows:

$$
\beta=\left(1+\frac{x}{L}\right)^{n}
$$

where $n=1,2$, or 3 .

The soil pressure profiles are shown in Figure 6.

The first three buckling loads were determined for each case as shown in Table 4. It is observed that the buckling loads experienced an increase with the increase in the exponent $(n)$. This is reasonable because, as shown in Figure 6, the area under the soil pressure distribution increases as power $n$ increases.

The mode shapes are shown in Figure 7. The first mode shape shifts to the left as the exponent increases, which is expected as the relative soil stiffness is less in that area.

5.2. Example 2: Crack Propagation. In this example, a simply supported column was analyzed. As the maximum moment in simply supported structural elements develops at the midspan, it was assumed that crack initiation occurred at the center of the column. The crack was modeled by a change in the cross-section of column, making it nonprismatic, as shown in Figure 8. This change was attained by reducing the moment of inertia $(I)$. The propagation of the cracks in the column was modeled by increasing the cracked length $\left(L_{\mathrm{cr}}\right)$. The following constants were used for the columns in this example: $E=1, I=1, L=1$, and $\beta=0$. The matrix of the columns to be modeled in this example was generated by varying the crack length to column length ratio $\left(L_{\mathrm{cr}} / L\right)$ and moment of inertia of the cracked section to gross moment of inertia $\left(I_{\mathrm{cr}} / I\right)$ ratio. The varying parameters from which the combinations were generated are shown in Table 5.

The analysis was performed, and the results for the columns are shown in Table 6. For each moment of inertia ratio, the first column shows the buckling load obtained, 
TABLE 2: Results for Case 3.

\begin{tabular}{|c|c|c|c|c|c|c|c|c|c|c|c|}
\hline \multirow{2}{*}{$k_{a}$} & \multirow{2}{*}{$k_{b}$} & \multicolumn{5}{|c|}{ Sway not permitted } & \multicolumn{5}{|c|}{ Sway permitted } \\
\hline & & $G_{a}$ & $G_{b}$ & $P_{\mathrm{cr}} / P_{E}($ program $)$ & $P_{\mathrm{cr}} / P_{E}$ (charts) & \% Error & $G_{a}$ & $G_{b}$ & $P_{\mathrm{cr}} / P_{E}($ program $)$ & $P_{\mathrm{cr}} / P_{E}$ (charts) & $\%$ error \\
\hline 40 & 20 & 0.05 & 0.1 & 3.471079 & 3.467779 & $0.095 \%$ & 0.15 & 0.3 & 0.86597 & 0.865333 & $0.074 \%$ \\
\hline 10 & 10 & 0.2 & 0.2 & 2.853986 & 2.85336 & $0.022 \%$ & 0.6 & 0.6 & 0.69959 & 0.700268 & $-0.097 \%$ \\
\hline 2 & 2 & 1 & 1 & 1.668095 & 1.669237 & $-0.068 \%$ & 3 & 3 & 0.29998 & 0.300244 & $-0.087 \%$ \\
\hline 0.4 & 0.1 & 5 & 20 & 1.097091 & 1.096461 & $0.057 \%$ & 15 & 60 & 0.04668 & 0.046709 & $-0.057 \%$ \\
\hline
\end{tabular}

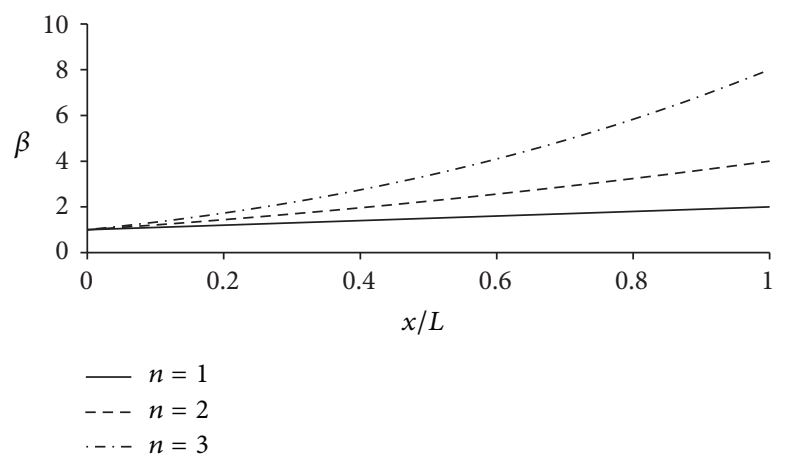

FIGURE 6: Soil pressure profiles for $n=1,2,3$.

TABle 3: Results for Case 4.

\begin{tabular}{lccc}
\hline \multicolumn{2}{c}{ Boundary conditions } & \multicolumn{2}{c}{ Critical load } \\
Strong end & Weak end & Eisenberger [2] & Proposed approach \\
\hline Free & Fixed & 3.1177 & 3.118548250634 \\
Fixed & Free & 4.1242 & 4.1238 \\
Hinged & Hinged & 14.5112 & 14.5105 \\
Hinged & Fixed & 29.449 & 29.4571 \\
Fixed & Hinged & 29.4788 & 29.4734 \\
Fixed & Fixed & 57.394 & 57.4019 \\
\hline
\end{tabular}

TABLE 4: Critical buckling loads for nonuniform elastic foundation example.

\begin{tabular}{lccc}
\hline$n$ & $P_{\mathrm{cr}}{ }^{1}$ & $P_{\mathrm{cr}}{ }^{2}$ & $P_{\mathrm{cr}}{ }^{3}$ \\
\hline 1 & 25.0219 & 43.3211 & 90.5166 \\
2 & 32.4221 & 45.9158 & 91.4651 \\
3 & 40.7501 & 53.5283 & 93.133 \\
\hline
\end{tabular}

TABLE 5: Cracking parameters for example 2.

\begin{tabular}{llccccc}
\hline$L_{\mathrm{cr}} / L$ & 0 & 0.1 & 0.2 & 0.3 & 0.4 & 0.5 \\
$I_{\mathrm{cr}} / I$ & 1 & 0.95 & 0.6 & 0.4 & - & - \\
\hline
\end{tabular}

while the second column shows the buckling load normalized against Euler's buckling load. Euler's buckling load is obtained for the uncracked prismatic column, which corresponds to a crack length ratio of 0 (row 1). The compression action in the column forces the crack to close; however, this causes an imperfection at that location which encourages the initiation of buckling from it.
In order to further analyze the obtained results, Figures 9 and 10 were plotted. Figure 9 plots the normalized buckling loads against the cracked span ratios. A reduction in the buckling load as the cracked span ratio increased was observed. This is logical as the propagation of the cracks reduces the overall moment of inertia of the column and thus decreases the load required to buckle the column. Each series of data points was fitted with a second-degree polynomial function. These regression equations provided excellent correlation $\left(R^{2}=0.99\right)$.

Figure 10 plots the normalized buckling loads against the cracked moment of inertia ratios. It was noted that the buckling load decreased as the cracked moment of inertia ratio decreased. This is rational because the smaller the ratio is, the larger the crack is and, thus, the smaller the overall moment of inertia becomes. An interesting trend observed was that the curve was bilinear for small cracked span ratios and approached the standard linear behavior as the span ratio increased.

5.3. Example 3: Column on Linearly Variable Elastic Foundation. In this example, a simply supported column was analyzed under different distributions of linear soil pressure. Details on the analyzed soil stiffness functions are shown in Table 7 and Figure 11. These functions provide a constant area under the soil stiffness profile and only differ in the distribution of that area. For this example the length $(L)$ was taken to be 10 .

In order to facilitate the analysis, a few parameters will be defined. The slope of the soil stiffness profile $\left(\beta^{\prime}\right)$ is calculated by taking the derivative as follows:

$$
\beta^{\prime}=\frac{d \beta(x)}{d x} .
$$


TABLE 6: Critical buckling loads and their normalized values for example 2.

\begin{tabular}{lcccccc}
\hline$I_{\mathrm{cr}} / I$ & & 0.95 & & 0.6 & & 0.4 \\
$L_{\mathrm{cr}} / L$ & $P_{\mathrm{cr}}$ & & $P_{\mathrm{cr}} / P_{e}$ & $P_{\mathrm{cr}}$ & $P_{\mathrm{cr}} / P_{e}$ & $P_{\mathrm{cr}}$ \\
\hline 0 & 9.869599 & 1 & 9.869599 & 1 & 9.869599 \\
0.1 & 9.767448 & 0.98965 & 8.695862 & 0.881075 & 7.53605 \\
0.2 & 9.67209 & 0.979988 & 7.806693 & 0.790984 & 6.148034 \\
0.3 & 9.58769 & 0.971437 & 7.157659 & 0.725223 & 5.29744 \\
0.4 & 9.517094 & 0.964284 & 6.693711 & 0.678215 & 4.755724 \\
0.5 & 9.461763 & 0.958678 & 6.370312 & 0.645448 & 4.405123 \\
\hline
\end{tabular}
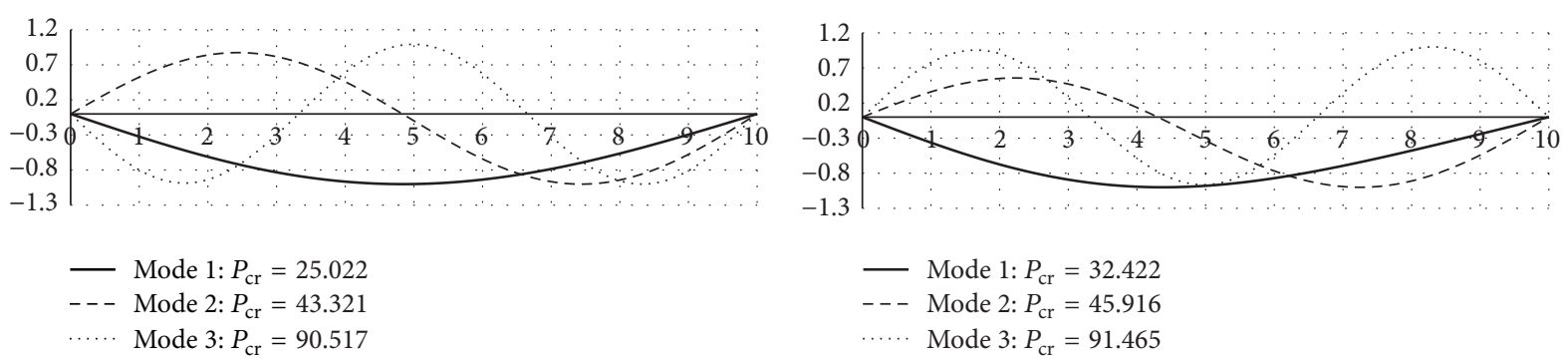

(a)

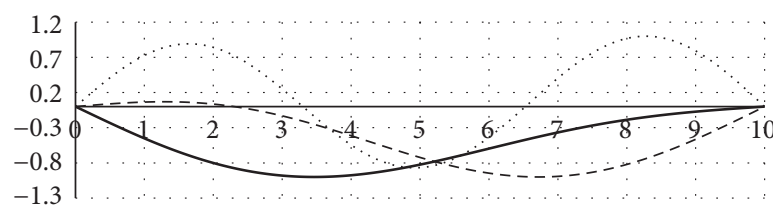

- Mode 1: $P_{\text {cr }}=40.75$
- - Mode 2: $P_{\text {cr }}=53.528$
… Mode 3: $P_{\text {cr }}=93.133$

(c)

FIgURE 7: Mode shapes for (a) $n=1$; (b) $n=2$; (c) $n=3$.

TABLE 7: Soil stiffness functions for example 3.

\begin{tabular}{lcc}
\hline Case \# & $\beta(x)$ & Type \\
\hline 1 & 3 & Uniform (rectangular) \\
2 & $2.25+0.15 x$ & Trapezoidal \\
3 & $1.5+0.3 x$ & Trapezoidal \\
4 & $0.75+0.45 x$ & Trapezoidal \\
5 & $0.6 x$ & Triangular \\
\hline
\end{tabular}

Another parameter is Kappa $(\kappa)$, which relates the uniform soil stiffness $\left(\beta_{1}\right)$ to the stiffness of the column $(E I)$ according to the following equation:

$$
\kappa=\sqrt[4]{\frac{\beta_{1}}{4 E I}} .
$$

For this example, columns with $\kappa$ values that correspond to the following $\beta / E I$ ratios were analyzed: $0.1,1,10,100$, and 200. The modulus of elasticity and moment of inertia were varied based on this ratio. The obtained buckling loads were normalized against Euler's buckling load and are shown in Table 8.
Additionally, the data points were plotted in Figures 12 and 13 . Figure 12 plots the normalized buckling load versus $\kappa$. It is observed that as the value of $\kappa$ increased, the normalized buckling load increased. Also, at low $\kappa$ values, which indicates that the EI is relatively high compared to $\beta$, the soil stiffness distribution did not have a significant effect on the buckling load. On the contrary, columns resting on relatively stiff soil experienced a significant variation in the buckling load based on the soil stiffness distribution (about 51\%). Data in Table 8 show that as the slope $\left(\beta^{\prime}\right)$ increases, the buckling load decreases. Figure 13 plots the normalized buckling load versus $\beta^{\prime}$. Lower $\kappa$ results are represented by straight lines, which indicate again that the distribution of soil stiffness did not significantly affect the buckling load for these columns. As $\kappa$ increased, the decrease in the critical load became more evident with the increase in $\beta^{\prime}$.

\section{Conclusion}

In this paper, an approach to determine buckling loads and their associated mode shapes semianalytically for nonprismatic columns with variable boundary conditions resting on nonuniform elastic foundation was formulated. An exact 
TABLE 8: Normalized buckling loads for example 3.

\begin{tabular}{lccccc}
\hline Case & 1 & 2 & 3 & 4 & 5 \\
$\beta^{\prime}$ & 0 & 0.15 & 0.3 & 0.45 & 0.6 \\
$\kappa$ & & & $P_{\mathrm{cr}} / P_{e}$ & 3.865672 & 3.746269 \\
\hline 0.294283096 & 4.054726368 & 4.034826 & 3.965174 & 10.01508 & 9.140704 \\
0.52331757 & 11.75376884 & 11.42211 & 10.78894 & 27.47236 & 22.9799 \\
0.930604859 & 35.42211055 & 33.97487 & 31.13065 & 77.44221 & 57.70352 \\
1.65487546 & 112.4170854 & 104.1457 & 92.38693 & 106.3065 & 76.12563 \\
1.967989671 & 157.8341709 & 146.1256 & 128.598 & & \\
\hline
\end{tabular}

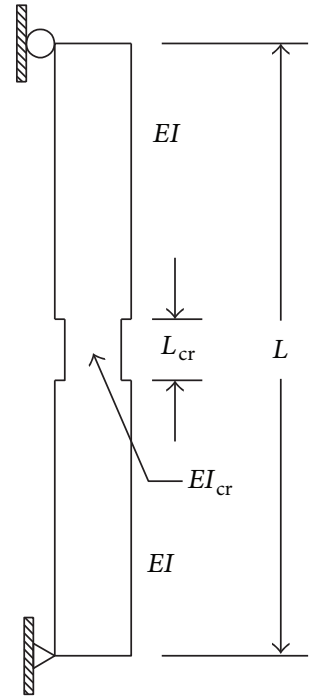

Figure 8: Column elevation for example 2.

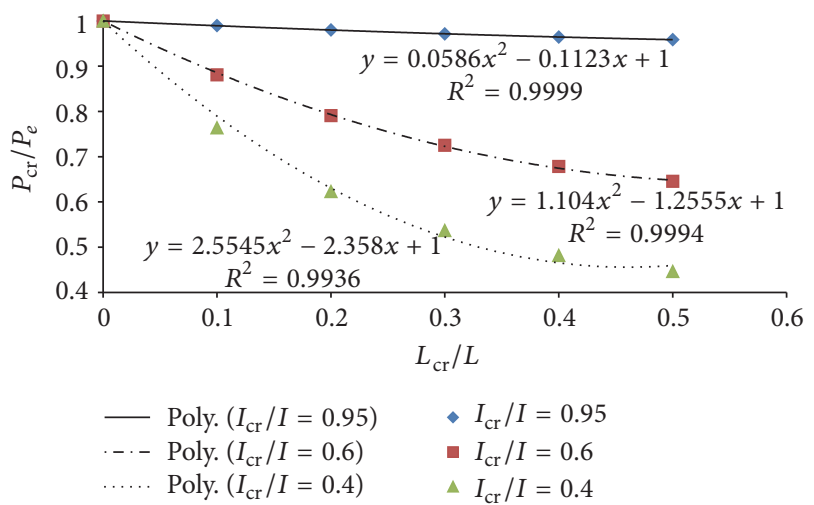

FIGURE 9: Normalized buckling load versus cracked span ratio for example 2 .

solution for the segment buckling equation was derived under all possible cases that govern the differential equation. Coupling the solutions of the various segments resulted in a system of equations that can be used to numerically solve for the buckling load and plot the mode shape. Sensitivity analysis and benchmarking verification of the derived solution is performed next. Finally, three examples addressing

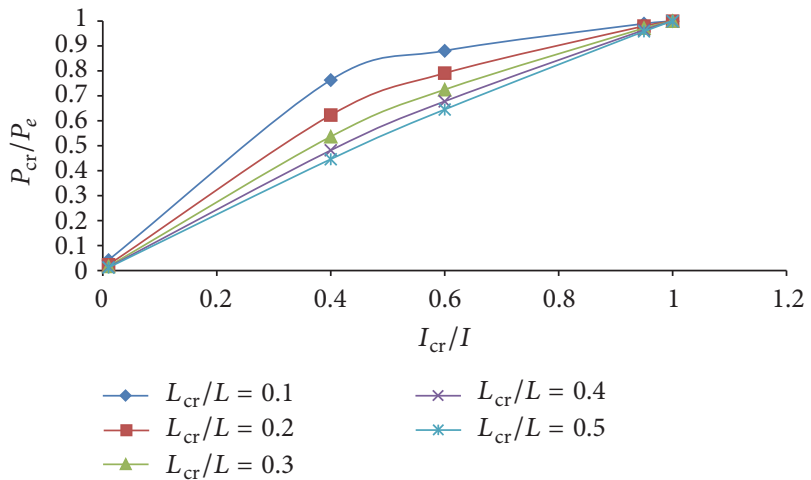

FIGURE 10: Normalized bucking load versus cracked moment of inertia ratio for example 2.

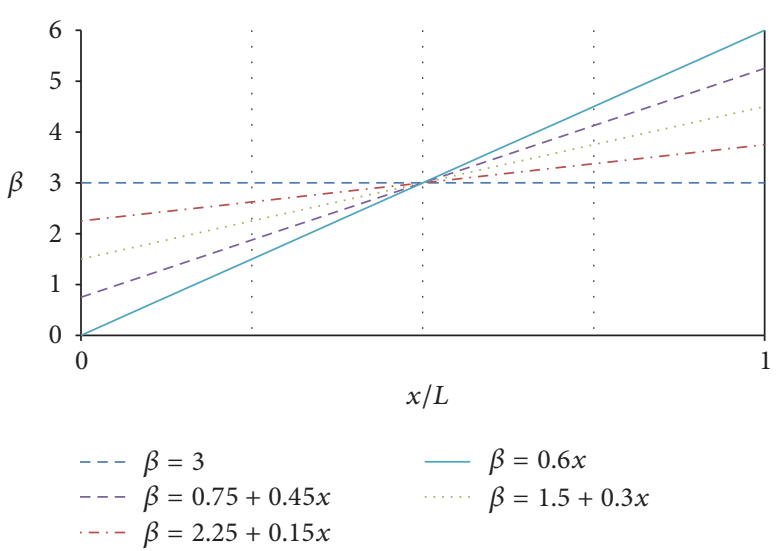

FIGURE 11: Soil stiffness profile along column span for example 3.

the comparison of linear and nonlinear elastic foundation, crack propagation using nonprismatic column analysis, and investigating the effect of changing soil stiffness distribution $\beta$ on the buckling load are examined.

\section{Conflicts of Interest}

The authors declare that there are no conflicts of interest regarding the publication of this paper. 


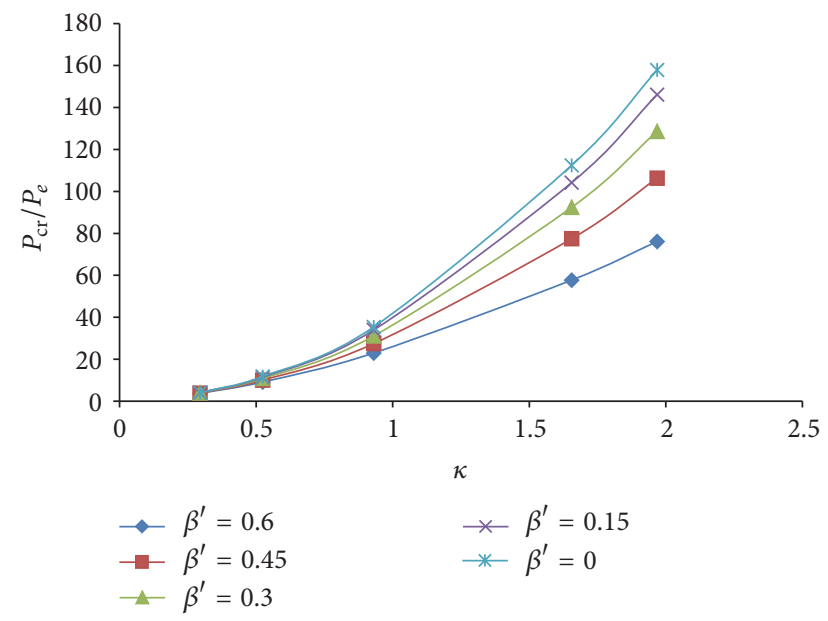

FIGURE 12: Normalized buckling load versus $\kappa$ for example 3.

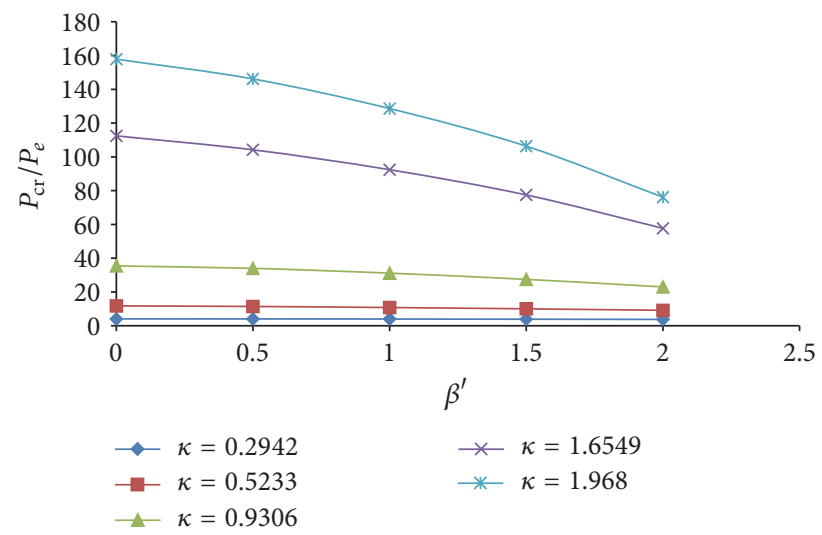

FIGURE 13: Normalized buckling load versus $\beta^{\prime}$ for example 3.

\section{Acknowledgments}

Publication of this article was funded in part by the Kansas State University Open Access Publishing Fund.

\section{References}

[1] L. G. Arboleda-Monsalve, D. G. Zapata-Medina, and J. D. Aristizabal-Ochoa, "Timoshenko beam-column with generalized end conditions on elastic foundation: dynamic-stiffness matrix and load vector," Journal of Sound and Vibration, vol. 310, no. 4-5, pp. 1057-1079, 2008.

[2] M. Eisenberger, "Buckling loads for variable cross-section members with variable axial forces," International Journal of Solids and Structures, vol. 27, no. 2, pp. 135-143, 1991.

[3] A. N. Kounadis and J. C. Ermopoulos, "Postbuckling analysis of a simple frame with varying stiffness," Acta Mechanica, vol. 54, no. 1-2, pp. 95-105, 1984.

[4] J. C. Ermopoulos, "Buckling of tapered bars under stepped axial loads," Journal of Structural Engineering, vol. 112, no. 6, pp. 1346$1354,1986$.

[5] S. B. Coskun, B. Öztürk, and S. B. Coşkun, Elastic Stability Analysis of Euler Columns Using Analytical Approximate Techniques,
Advances in Computational Stability Analysis, InTech, Rijeka, Croatia, 2012.

[6] S. B. Coşkun and M. T. Atay, "Determination of critical buckling load for elastic columns of constant and variable cross-sections using variational iteration method," Computers \& Mathematics with Applications, vol. 58, no. 11-12, pp. 2260-2266, 2009.

[7] B. Yang and D.-H. Park, "Exact buckling analysis of constrained stepped columns," International Journal of Structural Stability and Dynamics, vol. 3, no. 2, pp. 143-167, 2003.

[8] Y. Huang and X.-F. Li, "An analytic approach for exactly determining critical loads of buckling of nonuniform columns," International Journal of Structural Stability and Dynamics, vol. 12, no. 4, Article ID 1250027, 13 pages, 2012.

[9] B. K. Lee, S. J. Oh, and T. E. Lee, "Novel methods for calculating natural frequencies and buckling loads of columns with intermediate multiple elastic springs," International Journal of Structural Stability and Dynamics, vol. 2, no. 4, pp. 559-571, 2002.

[10] T. M. Atanackovic and B. N. Novakovic, "Optimal shape of an elastic column on elastic foundation," European Journal of Mechanics-A/Solids, vol. 25, no. 1, pp. 154-165, 2006.

[11] W. Lacarbonara, "Buckling and post-buckling of non-uniform non-linearly elastic rods," International Journal of Mechanical Sciences, vol. 50, no. 8, pp. 1316-1325, 2008.

[12] C. M. Wang, C. Y. Wang, and J. N. Reddy, Exact Solutions for Buckling of Structural Member, CRC Press, Boca Raton, Fla, USA, 2004.

[13] J. M. Gere and W. O. Carter, "Critical buckling loads for tapered columns," Journal of Structural Division ASCE, vol. 88, no. 1, pp. $1-11,1962$.

[14] M. Eisenberger and J. Clastornik, "Vibrations and buckling of a beam on a variable winkler elastic foundation," Journal of Sound and Vibration, vol. 115, no. 2, pp. 233-241, 1987. 


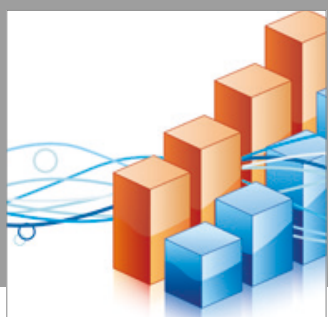

Advances in

Operations Research

vatersals

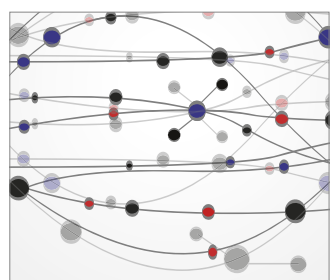

\section{The Scientific} World Journal
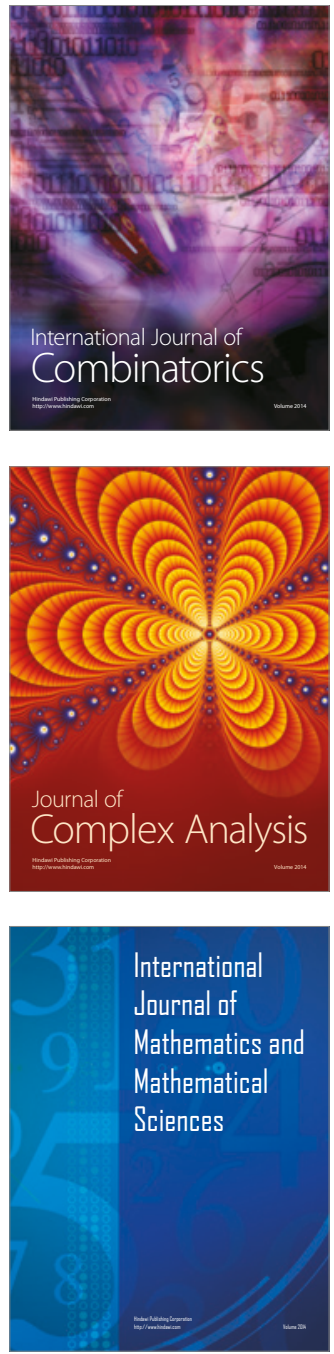
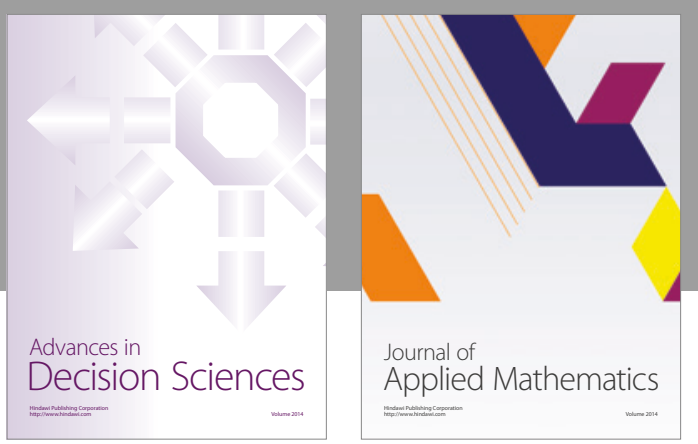

Algebra

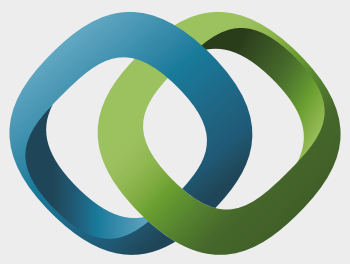

\section{Hindawi}

Submit your manuscripts at

https://www.hindawi.com
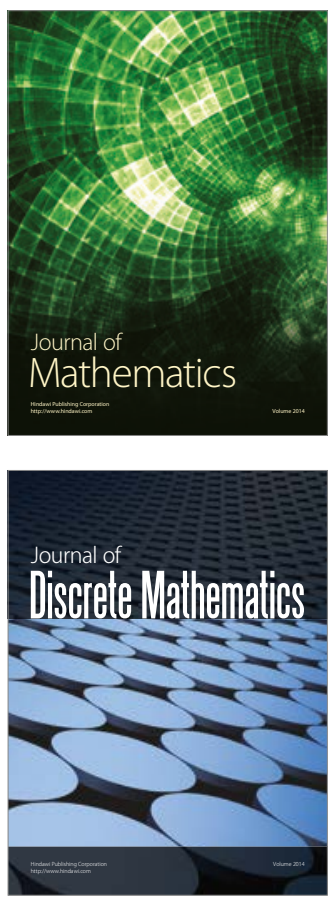

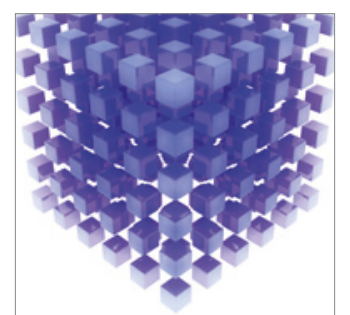

Mathematical Problems in Engineering
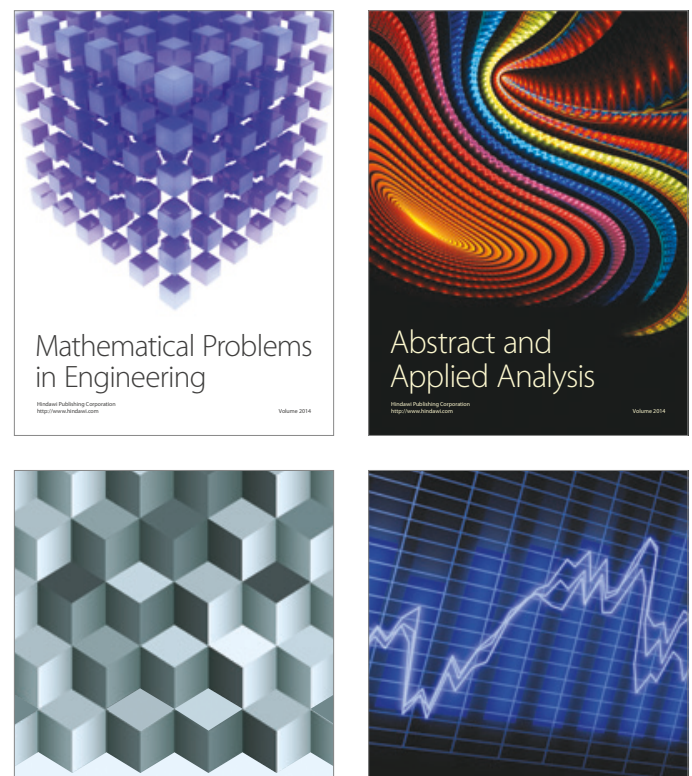

Journal of

Function Spaces

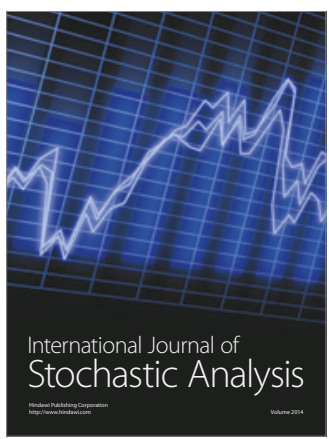

Probability and Statistics
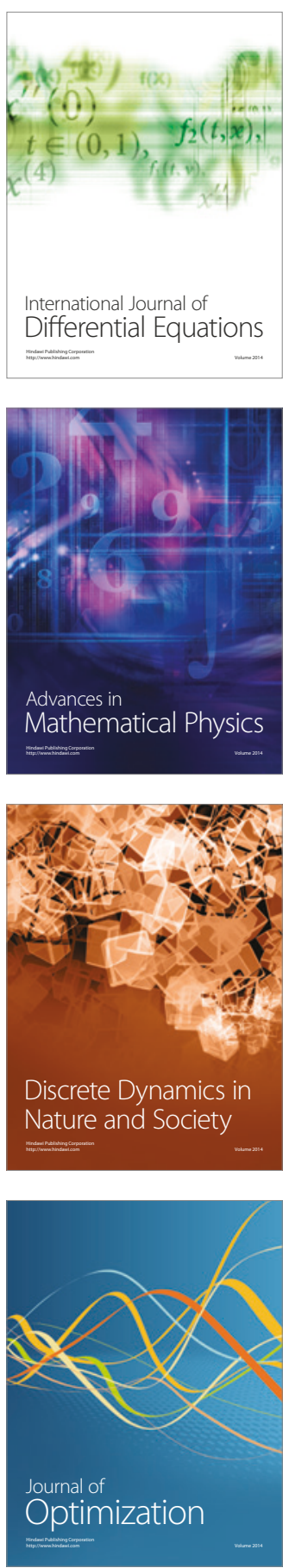